\title{
Natural occurrence of trichothecene-producing Fusaria isolated from India with particular reference to sorghum
}

\author{
S. V. Lincy $\cdot$ A. Chandrashekar $\cdot$ M. S. Narayan • \\ Rajan Sharma $\cdot$ R. P. Thakur
}

Received: 4 January 2010/Accepted: 14 August 2010/Published online: 29 August 2010

(C) Springer Science+Business Media B.V. 2010

\begin{abstract}
In this study a total of 167 isolates collected from different food materials (68.8\% from sorghum and the remaining from various other food materials) were assayed by PCR for amplification of the tri 5 gene present in trichothecene-producing Fusaria. Amplification of the tri 5 fragment was observed in 45 isolates (39 isolates from sorghum and 6 isolates from vegetables). Isolates found positive for presence of the tri 5 gene were classified into different morphological groups based on their cultural and conidial characters; 11 of the tri 5 positive isolates from moldy grains of sorghum, one from each morphology group were selected for further analyses. Five deoxynivalenol producers and three deoxynivalenol and Fusarenon-X producers were detected by analysing culture filtrates of the 11 isolates using GC-MS. One isolate each were identified as producers of NIV alone, or NIV along with DON or DAS toxins. Identification of these isolates to the species level was carried out using spore morphology and sequence comparison of the translation elongation factor 1-alpha (EF-1 $\alpha)$ gene against the database as well as using phylogenetic analyses. The isolates were identified as Fusarium proliferatum (6), F. nelsonii (2), F. equiseti (1), $F$. thapsinum (1) and F. sacchari (1). Amplified Fragment Length Polymorphism (AFLP) based grouping clustered the isolates of same species together. This is the first
\end{abstract}

S. V. Lincy - A. Chandrashekar · M. S. Narayan

Plant Cell Biotechnology Department, Central Food

Technological Research Institute, Mysore, Karnataka 570 020,

India

R. Sharma $(\bowtie) \cdot$ R. P. Thakur

International Crops Research Institute For the Semi-Arid

Tropics, Patancheru, Hyderabad, Andhra Pradesh 502 324, India

e-mail: r.sharma@cgiar.org detailed study of trichothecene production by Fusarium spp. associated with sorghum grain mold in India and the identification of $F$. nelsonii and $F$. thapsinum as producers of trichothecenes.

Keywords AFLP $\cdot$ EF- $1 \alpha \cdot$ Fusarium $\cdot$ Trichothecenes

\section{Introduction}

Many species of Fusarium infect economically important cereals like maize, wheat, rice, sorghum, barley, rye and oats (Placinta et al. 1999; Bottalico and Perrone 2002; Thakur et al. 2006). Fumonisins and trichothecenes, the toxins produced by Fusarium spp., are associated with many plant, animal and human diseases. Trichothecenes have significant phytotoxic activity and play an important role in animal pathogenesis (Placinta et al. 1999; Larsen et al. 2004). Fusarium is known to produce only group A (e.g. T-2, hydroxy-T-2, diacetoxyscirpenol and neosolaneol that does not have keto group at C-8) and group B (e.g. deoxynivalenol, 3-acetyldeoxynivalenol, 15-acetyl-deoxynivalenol, nivalenol and fusarenon- $X$ that possess a keto group at C-8) toxins among the four groups of trichothecenes that have been classified based on the differential substitution of the side chains in the trichothecene skeleton (Wannemacher et al. 2000).

Deoxynivalenol (DON), followed by nivalenol (NIV), diacetoxyscirpenol (DAS) and T-2, are the most prevalent trichothecene toxins produced by Fusarium spp. infecting wheat and barley (Placinta et al. 1999; Bottalico and Perrone 2002). Adejumo et al. (2007) have reported trichothecene production in Fusarium isolates from maize. DON and NIV have been found as contaminants of sorghum in Ethiopia (Ayalew et al. 2006). Data for occurrence of 
Fusarium toxins in India has implicated fumonisins and zearalenone (ZEA) in maize as the major cause of toxicosis (Bhavanishankar and Shantha 1987; Sinha 1990; Bhat et al. 1997; Janardhana et al. 1999). There have been reports for the presence of trichothecenes in sorghum and wheat from India (Rukmini and Bhat 1978; Bhavanishankar and Shantha 1987; Bhat et al. 1989). Saharan et al. (2007) have reported variability among $F$. graminearum isolates from various parts of India. These researchers however did not report the toxins produced by this fungus. The gene tri 5 codes for the enzyme trichodiene synthase (TS) (EC 4.2.3.6) that is commonly involved in the synthesis of all Fusarium trichothecenes (Desjardins et al. 1993). This work is the result of screening of Fusarium isolates from various Indian foods by PCR for the identification of isolates positive for the presence of the tri 5 gene. Various techniques such as GC, HPLC and GC-MS for the identification of trichothecene toxins were employed. Further investigation was carried out into morphological and molecular characterization of toxin-producing isolates from sorghum for their identification at the species level.

\section{Materials and methods}

Fungal strains and their maintenance

Of the 167 Fusarium isolates used in this study, 115 were isolated from moldy grains of different genotypes of sorghum. The remaining strains were isolated from different food materials such as maize, pigeon-pea, coffee, ginger and vegetables such as beans (Phaseolus vulgaris), bitter gourd (Momordica charantia), chocho (Sechium edule), cauliflower (Brassica oleracea botrytis) and red chilli (Capsicum annum). The cultures were purified by single spore culture. One hundred grams of material were collected, ground to fine powder and mixed properly. To $9 \mathrm{ml}$ sterile water, $1 \mathrm{~g}$ of the ground food material was added and mixed properly to obtain a uniform suspension. Successive serial dilutions were prepared by transferring $1 \mathrm{ml}$ each of the suspensions to fresh tubes containing $9 \mathrm{ml}$ sterile water (1:10 dilution obtained at each transfer), until 1-10 conidia were observed in a drop of the suspension when viewed under microscope at $100 \times$ magnification. $100 \mu \mathrm{l}$ of such suspensions were plated on to potato dextrose agar (PDA, HiMedia, Mumbai, India) and the plates were incubated at $28^{\circ} \mathrm{C}$ overnight. Bacterial contamination during isolation of Fusarial cultures from food materials were avoided by including streptomycin at a concentration of $50 \mu \mathrm{g} / \mathrm{ml}$ in the medium while plating. Isolation of germinated conidia in the plates was carried out under dissection microscope, where the hyphae appeared as small threads on the agar surface. Using a sterile scalpel the agar piece bearing the hyphae was excised and was placed on to fresh PDA plate with the help of sterile nichrome wire. The plates were incubated at $28^{\circ} \mathrm{C}$ for $3-5$ days till a profuse matlike growth was observed on the agar surface. The purified Fusarium isolates were subcultured on to PDA slants and maintained on PDA at $4^{\circ} \mathrm{C}$. NCIM 651 (Gibberella saubinetti, renamed as $F$. asiaticum in the present study), a DON producer obtained from the National Collection of Industrial Micro-organisms (NCIM), Pune, India, was used as the standard culture.

\section{Culture conditions}

Isolates of Fusarium were grown on autoclaved rice (50 g) and incubated at $28^{\circ} \mathrm{C}$ for a period of 2 weeks prior to HPLC and GC-MS analysis of trichothecenes. Cultivation of Fusarium for GC analysis was carried out in $50 \mathrm{ml}$ GYEP ( $5 \%$ glucose, $0.1 \%$ yeast extract, $0.1 \%$ peptone) broth for a period of 2 weeks at $28^{\circ} \mathrm{C}$, without shaking. The isolates were grown in potato dextrose broth with shaking at $180 \mathrm{rev} / \mathrm{min}$, at $28^{\circ} \mathrm{C}$ for a period of 4-5 days prior to isolation of genomic DNA.

Chemicals and reagents

Trichothecene standards were purchased from Sigma (St. Louis, MO, USA). T-2 and DAS stock solutions in ethyl acetate ( $1 \mathrm{mg} / \mathrm{ml}$ stock solution) and DON, NIV and Fus-X solutions in acetonitrile ( $1 \mathrm{mg} / \mathrm{ml}$ stock solution) were stored at $4^{\circ} \mathrm{C}$.

The PCR primers were procured from Sigma-Aldrich, Bangalore, India. Taq polymerase, XT-Taq, dNTPs, agarose and DNA marker (100 bp ladder) were purchased from Bangalore Genei, Bangalore, India. Other chemicals used in this study were of molecular biology grade and were purchased from Sigma-Aldrich (USA), Merck (Germany), SRL (Bangalore, India) and Bangalore Genei (Bangalore, India).

\section{Identification of toxigenic fungi by PCR}

Three- to four- days-old mycelium of the 167 Fusarium isolates grown in potato dextrose broth was harvested by filtration and washed in sterile deionized water. The mycelium was ground well in lysis buffer (50 mM Tris, pH-8; 50 mM EDTA, pH-8; 3\% SDS, $1 \% \quad \beta$-mercaptoethanol) which was preheated at $65^{\circ} \mathrm{C}$ for $90 \mathrm{~min}$. The tubes were incubated for $60 \mathrm{~min}$ in a water bath set at $65^{\circ} \mathrm{C}$ and vortexed intermittently at intervals of $15 \mathrm{~min}$. An equal volume of a phenol: chloroform: isoamyl alcohol mixture $(25: 24: 1)$ was added and the tubes were centrifuged at 10,000 rev/min for $15 \mathrm{~min}$. The supernatant was transferred to a fresh micro centrifuge tube and re-extracted 
with phenol: chloroform: isoamyl alcohol 3-4 times, which helped in minimizing protein contamination. $200 \mu \mathrm{l}$ of $1 \mathrm{M}$ $\mathrm{NaCl}$ and $800 \mu \mathrm{l}$ of ice cold ethanol were added and the tubes were incubated overnight at $-20^{\circ} \mathrm{C}$ for precipitation of DNA. DNA was collected by centrifugation at $10,000 \mathrm{rev} / \mathrm{min}$ for $15 \mathrm{~min}$ and the pellet obtained was washed in $70 \%$ ice-cold ethanol. DNA was finally suspended in suitable volume of TE buffer and stored at $-20^{\circ} \mathrm{C}$.

Forward and reverse primers designed for the amplification of part of the tri 5 gene were used for the detection of trichothecene producers. Three primers designated as T5GF2 (5'-ACCATCCTCCATTCACCAC-3'), T5GR2 (5'CACACCTCACCCTCCTTCT-3') (Lincy et al. 2008) and T5GR1 (5'-TYACTCCACTAGCTCAATTG- $3^{\prime}$ ) were used for the detection of the tri 5 gene. T5GF2-T5GR1 primers were expected to amplify a fragment of size $652 \mathrm{bp}$ and the T5GF2-T5GR2 primer sets to amplify a 379 bp fragment from trichothecene producers. EF1 (5'-ATGGGTAAGGARGACAAGAC-3') and EF2 (5'-GGARGTACCAGTSATCATGTT-3') primers (O’Donnel et al. 1998) were used to amplify $\sim 650 \mathrm{bp}$ fragment, a part of the translation elongation factor- $1 \alpha(\mathrm{EF}-1 \alpha)$ gene of Fusarium. PCR was performed in a $25 \mu \mathrm{l}$ final reaction mixture containing $2.5 \mu 110 \times$ buffer, $1 \mathrm{U}$ of Taq DNA polymerase or XT-Taq (in case of EF1-PCR for sequencing), $0.5 \mu \mathrm{d} \mathrm{dNTP}$ mixture (2.5 mM each), $2 \mu$ template DNA (20-50 ng) and $1 \mu \mathrm{l}$ each of both forward and reverse primers (10 nM). DNA amplification was performed in a thermocycler (Gene Amp PCR system 9700, Perkin-Elmer, USA) using an initial 5 min denaturation at $95^{\circ} \mathrm{C}$ followed by 34 cycles of $1 \mathrm{~min}$ denaturation at $95^{\circ} \mathrm{C}, 50 \mathrm{~s}$ annealing at 55 and $50^{\circ} \mathrm{C}$ for tri 5 and EF1 primers, respectively, and $1 \mathrm{~min}$ extension at $72^{\circ} \mathrm{C}$ followed by a final extension of $10 \mathrm{~min}$ at $72^{\circ} \mathrm{C}$. The PCR amplification products were checked on $1.5 \%$ agarose gel electrophoresis. The EF- $1 \alpha$ PCR amplified products were sequenced using the EF1 primers.

\section{Morphology and conidial characteristics}

Cultural characteristics such as colony color and nature of mycelial growth were noted after growth on PDA from 1 week-old cultures of the 45 isolates positive for tri 5 gene. Conidial features such as presence of macro- and microconidia and their shape were noted after growth on low nutrient banana leaf agar (BLA, prepared by adding sterile banana leaf pieces in $1.5 \%$ tap water agar). Mycelial suspensions of the isolates $(100 \mu \mathrm{l}$ each) were plated on to BLA and incubated at $28^{\circ} \mathrm{C}$ for 2 weeks to allow sporulation. The spores were suspended in sterile deionized water and observed under a Phase Contrast Microscope (Olympus BX40 Olympus Optical Co. Ltd., Japan). The identification system for Fusarium proposed by Leslie and
Summerell (2006) was adopted for grouping the PCR positive isolates.

\section{Detection of T-2 toxin}

T-2 toxin was extracted from the GYEP broth culture filtrates of the 11 Fusarium isolates as described by Tag et al. (2001). The culture filtrate was collected by filtration and stored at $-20^{\circ} \mathrm{C}$. Prior to $\mathrm{GC}$ analysis $5 \mathrm{ml}$ of the filtrate was mixed with an equal volume of ethyl acetate. The mixture was centrifuged at 3,000 rev/min for $10 \mathrm{~min}$ and the upper phase was collected. This was concentrated by heating in a boiling water bath (in presence of pumice stones to prevent spillage) till a final volume of $100 \mu \mathrm{l}$ was obtained. Ethyl acetate $(500 \mu \mathrm{l})$ was then added. The resuspended residue $(5-10 \mu \mathrm{l})$ was then injected into the gas chromatograph (Shimadzu Gas Chromatograph GC$15 \mathrm{~A}$ provided with a FID detector). GC analyses were performed on a SE-30 column $(3 \mathrm{~m} \times 3 \mathrm{~mm}$ id, mesh size $80 / 100$, ageing temperature $280^{\circ} \mathrm{C}$ ). Injector and detector temperatures were 260 and $270^{\circ} \mathrm{C}$, respectively. The oven temperature was programmed from $100^{\circ} \mathrm{C}$ (held for one $\min$ ) to $280^{\circ} \mathrm{C}$ (held for $10 \mathrm{~min}$ ) at the rate of $10^{\circ} \mathrm{C} / \mathrm{min}$. Nitrogen was used as carrier gas with a flow rate of one $\mathrm{ml} /$ min. Retention time of the toxin from the fungal isolates was compared with that of the standard.

\section{Detection of DON}

DON was detected using HPLC following the method of Martins and Martins (2001). Cultures of the 11 isolates were extracted with $250 \mathrm{ml}$ acetonitrile: water (84:16). The extracts were filtered through Whatman No. 1 filter paper, defatted with double the volume of $n$-hexane and the lower phase was extracted with equal volume of dichloromethane and chloroform. The lower phase was evaporated to dryness and stored at $-20^{\circ} \mathrm{C}$ until use. The residues were resuspended in $500 \mu \mathrm{l}$ of acetonitrile: water (84:16) and injected into the HPLC attached with $\mathrm{C}-18$ reverse phase column (Li Chrospher 100). The mobile phase used was acetonitrile: water in the ratio $84: 16$ at the rate of $1 \mathrm{ml} / \mathrm{min}$. The column was maintained at $28^{\circ} \mathrm{C}$. LC-10 AT Liquid Chromatograph (Shimadzu) provided with a SEL-10A VP Shimadzu UV-VIS detector was used and the results were recorded at $218 \mathrm{~nm}$. Retention time of the toxin from fungal isolates was compared with that of the standard.

\section{GC-MS}

The procedure for extraction of toxin as described by Onji et al. (1998) was followed. Ten $g$ of the rice cultures of the 11 isolates were extracted with $50 \mathrm{ml}$ acetonitrile and filtered. A $20 \mathrm{ml}$ volume of the filtrate was mixed with equal 
volume of $10 \%$ zinc acetate and allowed to stand at $28^{\circ} \mathrm{C}$ for $15 \mathrm{~min}$. $10 \mathrm{~g}$ Celite 545 was added to the mixture and filtered. The filtrate was mixed with $3 \mathrm{~g}$ of ammonium sulphate in a separating funnel. The aqueous layer was re-extracted with $30 \mathrm{ml}$ acetonitrile, mixed with double the volume of hexane and then allowed to stand for $10 \mathrm{~min}$ for separation of phase. The lower phase was mixed with equal volume of dichloromethane-chloroform $(1: 1)$. The toxin was adsorbed on a charcoal-alumina (1:4) column and eluted with $3 \mathrm{ml}$ of chloroform-methanol (1:4). The eluate was evaporated to dryness and stored at $4{ }^{\circ} \mathrm{C}$. The residue was finally dissolved in $1 \mathrm{ml}$ of acetone prior to GC-MS analysis.

GC-MS was carried out following the procedure of Onji et al. (1998). 2-5 $\mu$ l of the toxin extract was injected into the gas chromatograph (TurboMass Gold Mass Spectrometer provided with a MS detector, Perkin Elmer Instruments) along with toxin standards (5 $\mu \mathrm{l}$ from stock, redissolved in acetone). GC separations were performed on a SE-30 column $(30 \mathrm{~m} \times 0.25$ id $\times 0.25 \mu \mathrm{m}$ film thickness). Column oven temperature was maintained at $120^{\circ} \mathrm{C}$ for $0.2 \mathrm{~min}$, programmed from 120 to $250^{\circ} \mathrm{C}$ at the rate of $20^{\circ} \mathrm{C} / \mathrm{min}$. Nitrogen carrier gas was used at a constant flow rate of $1 \mathrm{ml} / \mathrm{min}$. Injector and detector temperatures were 260 and $270^{\circ} \mathrm{C}$ respectively. The mass conditions were as follows: full scan mode; ionization energy, $70 \mathrm{eV}$ : ion source temperature, $150^{\circ} \mathrm{C}$ and interface temperature, $150^{\circ} \mathrm{C}$.

\section{Phylogenetic analyses}

Sequences of the EF1-PCR fragments were searched against those in the FUSARIUM-ID v. 1.0 database (Geiser et al. 2004) using BLAST (Altschul et al. 1997) and also used in phylogenetic and molecular evolutionary analyses conducted using Molecular Evolutionary Genetics Analyis3.1 (MEGA version 3.1; Kumar et al. 2004). Neighbour Joining (NJ) analysis was performed using the heuristic search options with 1,000 parsimony bootstrap replications on DNA sequences of the EF- $1 \alpha$ gene for analysing similarity levels of isolates of the present study in relation to the already existing toxigenic species of Fusarium. Indels were coded as single events.

\section{AFLP Analysis}

AFLPs were generated based on selective amplification of DNA restriction fragments (Vos et al. 1995). The analysis was carried out using the AFLP core reagent kit and the AFLP starter kit of Gibco BRL ${ }^{\circledR}$ (Catalogue numbers 10544-013, 10483-014) (Life Technologies, USA) following the manufacturer's protocols with slight modifications. Primary template DNA was prepared in a one-step restriction-ligation reaction. Fungal genomic DNA (400 ng) was digested with EcoRI and MseI at $37^{\circ} \mathrm{C}$ for $2 \mathrm{~h}$ and heated at $70^{\circ} \mathrm{C}$ for $15 \mathrm{~min}$ to inactivate the enzyme. The DNA fragments were ligated to EcoRI and MseI adapters at $20^{\circ} \mathrm{C}$ for $2 \mathrm{~h}$. After terminating the reaction, the ligation mixture was diluted tenfold with TE buffer and the fragments were pre-amplified in a thermocycler (MJ Research, USA) using EcoRI (5'-GACTGCGTACCAATTC- $\left.3^{\prime}\right)$ and $M s e$ I (5'-GATGAGTCCTGAGTAA- $3^{\prime}$ ) primers. The cycle profile was as follows: $94^{\circ} \mathrm{C}$ for $30 \mathrm{~s}$, $56^{\circ} \mathrm{C}$ for $1 \mathrm{~min}$ and $72^{\circ} \mathrm{C}$ for $1 \mathrm{~min}$, in a total of 30 cycles. For selective amplification, five EcoRI + Mse I AFLP primer combinations, each with two-base extension in EcoRI and three-base extension in $M S e I$ primer at $3^{\prime}$ end (E$\mathrm{tg}+\mathrm{M}$-cag, E-aa + M-ctt, E-ac + M-cag, E-tc + M-ctt and E-tg + M-cag), were examined in the 11 isolates. The EcoRI primer was labeled with $\left[\gamma_{-}-{ }^{32} \mathrm{P}\right]-\mathrm{ATP}(3,000 \mathrm{Ci} /$ mmol) and the selective amplification was carried out according to the manufacturer's protocol. After selective amplification, the PCR products in $3 \mu$ sub-samples were separated by electrophoresis on $6 \%$ denaturing polyacrylamide DNA sequencing gel containing 7.5 M urea. Autoradiograms were obtained using Kodak X-Omat film.

AFLP profiles of 11 isolates were used to construct a binary matrix. Each band was scored as present (1) or absent (0) across the isolates. The data were then analysed using Numerical Taxonomy System Version 2.2 (NTSYSpc). The proximity matrix was computed using Dice similarity coefficient and a dendrogram was constructed by unweighted pair group method of arithmetic averages (UPGMA) using the SAHN (Sequential Agglomerative Hierarical Nested) cluster analysis module (Rohlf 2000).

\section{Results and discussion}

Analysis of trichothecenes in Fusarium isolates

In this study PCR with primers capable of amplifying 652 (using primers T5GF2-T5GR1) and 379 bp (using nested primers T5GF2-T5GR2) fragments of the tri 5 gene were used initially to distinguish the isolates that could produce trichothecene toxins. A total of 45 isolates (39 isolates from sorghum and six from other food materials) were thus identified as positive for tri 5 (Fig. 1). Reliability of PCR was further confirmed by the negative reactions that were obtained when Fusaria other than those that produced trichothecenes or when fungi other than Fusaria were used in PCR (data not shown). Similar group specific PCR assay for the detection of trichothecegenic Fusaria have been developed by Niessen and Vogel (1998) using primers specific for tri 5 . 


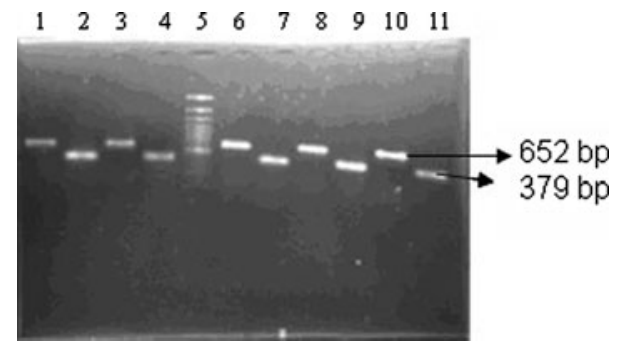

Fig. 1 PCR amplification of 652 and 379 bp tri 5 fragments from the Fusarium isolates. Lanes. 1 and 2: ICR PQ-12; 3 and 4: ICR50; 5: $3 \mathrm{~Kb}$ DNA ladder; 6 and 7: ICR-PQ-2; 8 and 9: FM246; 10 and 11: ICR61

The 45 isolates found positive for tri 5-PCR were classified into different morphology groups based on mycelial growth and conidial features; out of which 11 isolates from sorghum (one each from the different morphology groups) were selected at random for profiling the toxin produced by them and their identification at the species level. Trichothecene production of the 11 isolates was confirmed through GC-MS analysis of these toxin extracts. The expected mass fragmentation patterns for the different trichothecenes given in Table 1 were compared with the MS spectra of standards. HPLC and GC-MS revealed presence of DON in culture filtrates of 9 of the 11 isolates while GC and GC-MS failed to detect T-2 and HT2 toxins in any of the 11 isolates studied. This is in concordance with the reports of Bhavanishankar and Shantha (1987) and Ramakrishna et al. (1989) that DON was secreted by Fusarium cultures isolated from sorghum. DON has also been identified as the major contaminant especially in wheat and wheat-based products, worldwide (Placinta et al. 1999; Bottalico and Perrone 2002; Larsen et al. 2004). DON alone was detected in the culture filtrates of five isolates designated as ICR-PQ-12, ICR-PQ-13, ICR11, ICR50 and FM242. Three isolates (ICR61, FM311 and ICR-PQ-2) produced Fus-X along with DON. Culture filtrate of the isolate ICR1 contained DAS and NIV whereas the isolate FM246 secreted DON and NIV. Production of only NIV was observed in the isolate ICR57. After analysis of extracts from ICR57 and FM246, we concluded that NIV was the major toxin present, though a peak for DON was apparent. Taking into consideration the chances that NIV was the major trichothecene formed, the degraded product DON was not taken into consideration as reported by Onji et al. (1998). NIV, whose $\mathrm{m} / \mathrm{z}$ ratio, though small as compared to that of DON, was clearly present. A similar explanation may be given for our reporting the presence of Fus-X. NIV is a degradation product of Fus-X. Fus-X was present and so NIV, the degradation product of Fus-X was not given importance. Different trichothecenes or toxigenic Fusarium strains have been encountered in various food and feed items like barley (Bottalico and Perrone 2002; Ayalew et al. 2006), maize (Schollenberger et al. 2006; Adejumo et al. 2007) oats and other food and feed materials (Langseth and Elen 1996; Prasad et al. 2000; Schollenberger et al. 2006). Adejumo et al. (2007) have reported trichothecene production in Fusarium isolates from maize. DON and NIV have been found as contaminants of sorghum in Ethiopia (Ayalew et al. 2006). The present study also revealed that the Fusarium spp. associated with sorghum grain mold in India is mostly capable of producing DON. In contrast, Rukmini and Bhat (1978) and Bhavanishankar and Shantha (1987) have detected T-2 toxin in sorghum. We have also reported the presence of the type A trichothecenes, T-2 and DAS in sorghum (Lincy et al. 2008). Janardhana et al. (1999) has reported contamination of maize samples from Karnataka (India) with T-2 and DON whereas the presence of T-2 toxin, DON and NIV has been reported from Kashmir (India) in bread made from moldy wheat (Bhat et al. 1989).

\section{Characterization of Fusarium species using} morphology, EF- $1 \alpha$ gene sequences, phylogenetic and molecular evolutionary analyses, AFLP and toxin production

Eleven isolates that were identified as positives for tri 5-PCR and ability to produce trichothecenes were characterized to species level following a study of colony and spore features, sequences of the EF- $1 \alpha$ gene and AFLP. Growth pattern on PDA varied from profuse velvety mycelia to sparsely growing cotton-threadlike mycelia (Table 2). Conidial features, studied after growth on BLA, revealed clear differences in the structure, shape and number of septa of macro- and microconidia. Few isolates produced profuse macroconidia along with septate or aseptate microconidia, whereas in some isolates (the majority of which were from sorghum) only microconidia were observed (Table 2). Specific primers for EF-1 $\alpha$ gene
Table 1 Ions monitored for Fusarium trichothecene determination by GC-MS

\begin{tabular}{lll}
\hline Trichothecene & Molecular wt. & Daughter ions used for identification \\
\hline NIV & 312 & $312,253,235,205,177,187,159,149,121,105$ \\
DON & 296 & $296,281,275,259,235,207,181,165,147,129$ \\
DAS & 366 & $366,282,273,266,249,243,236,254,224,208,196,184$ \\
Fus-X & 354 & $355,325,308,284,281,264,256,241,207,191,185,171$ \\
\hline
\end{tabular}




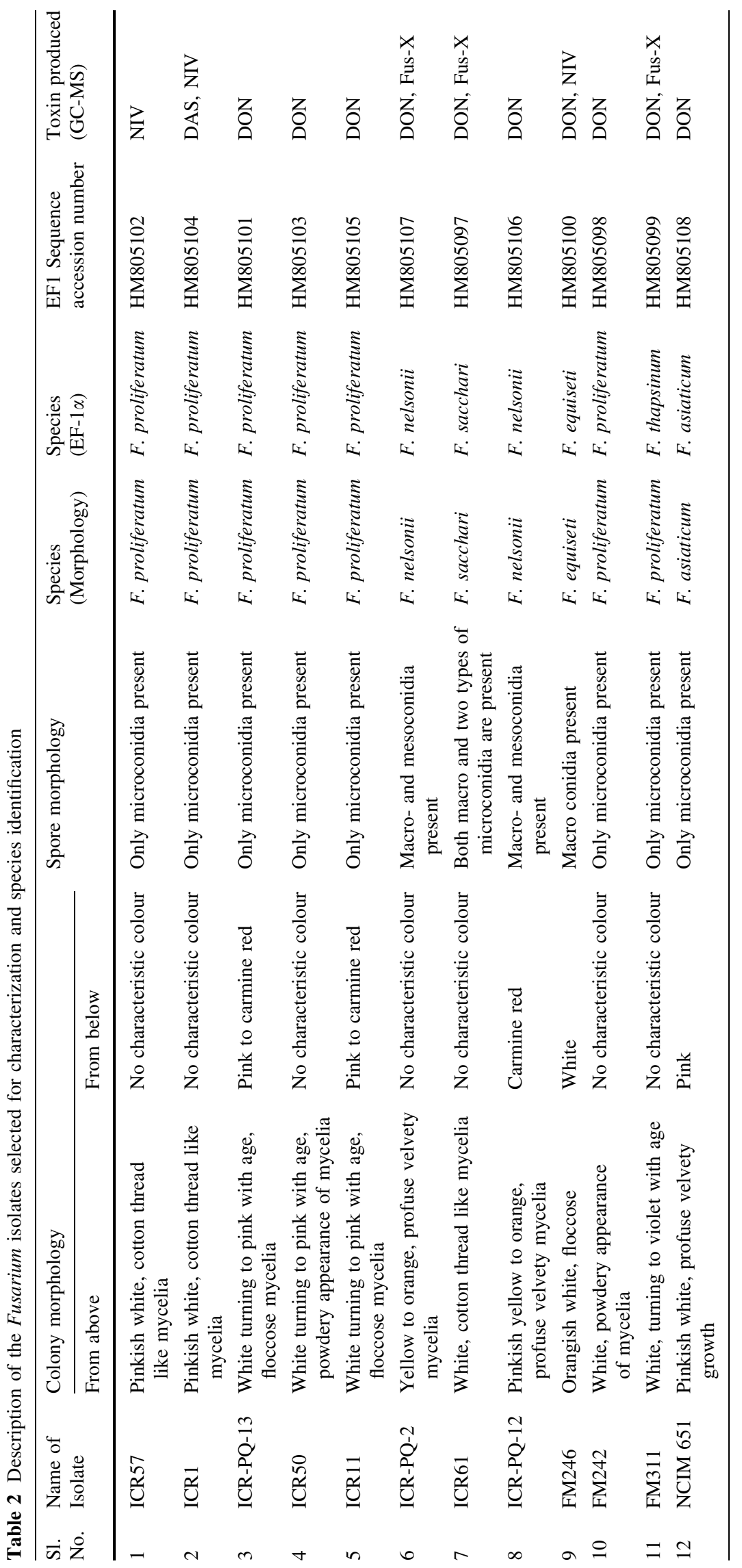




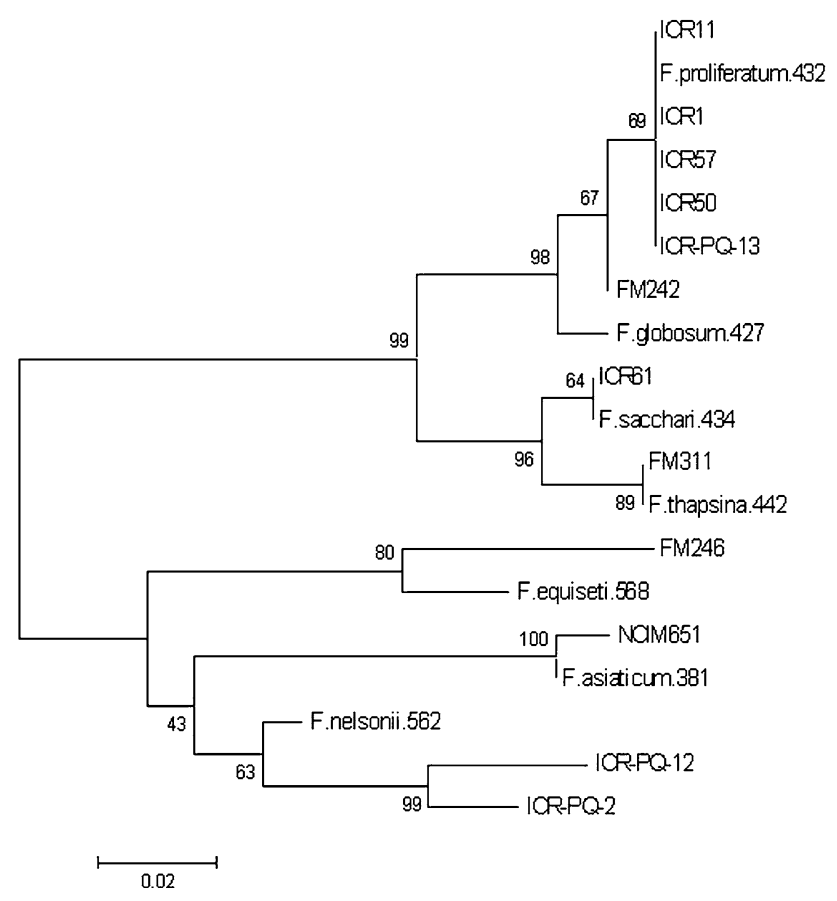

Fig. 2 Neighbour Joining consensus tree for translation elongation factor $-1 \alpha(E F-1 \alpha)$ sequences of Fusarium isolates used in the study. The numbers in the control strains indicates the sequence ID in the FUSARIUM-ID v. 1.0 database

amplified a $\sim 650 \mathrm{bp}$ fragment. The amplified area spanned 3 introns. These introns are highly informative, allowing identification to the species level. The sequences of EF-1 $\alpha$ gene from each of the 11 Fusarium isolates (query) and the standard culture F. asiaticum (NCIM 651) were compared to those from various known species of Fusarium (subject). Similarity of the query sequence to those present in the database was the criterion used for the identification of species (Fig. 2). The sequences have been submitted in the NCBI GenBank database and the accession numbers are provided in Table 2. A high level of polymorphism was observed among isolates following selective amplification with 5 AFLP primer combinations $($ Etg + Mcag, Eaa + Mctt, Eac + Mcag, Etc + Mctt and Etg + Mcag) (Fig. 3). A total of 338 bands were amplified from 5 primer combinations, of which 331 were polymorphic. The dendrogram generated from the AFLP data revealed genetic similarity among the isolates of same species by clustering them together (Fig. 4).

All the isolates that produced only microconidia (ICR1, ICR11, ICR-PQ-13, ICR50, ICR57, FM242 and FM311) were grouped as $F$. proliferatum. The EF1 sequences for part of the gene that was amplified by the primers EF1-EF2 from the isolates ICR1, ICR11, ICR-PQ-13, ICR50 and ICR57 were completely identical to that of $F$. proliferatum, a species included in Liseola section of Fusarium (Leslie et al. 2005). These isolates formed a single clade in both

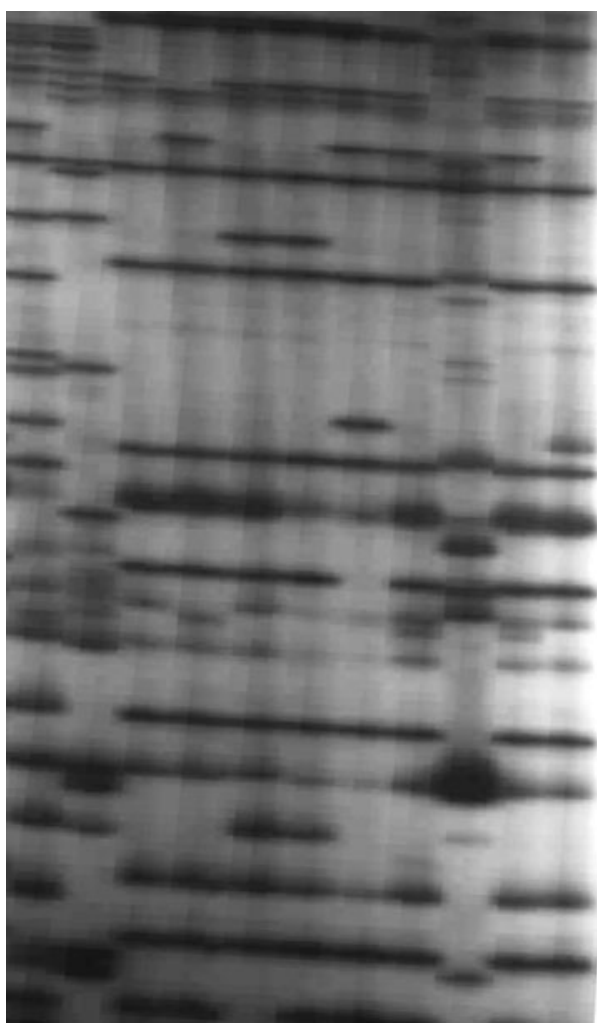

Fig. 3 AFLP fingerprint of 11 isolates of Fusarium spp. generated from selective amplification with E-tc + M-ctt primer combination

AFLP and molecular phylogenetic analysis (Figs. 2, 4). The isolates ICR11, ICR-PQ-13 and ICR50 were identified as producers of DON (Table 2). ICR1 secreted NIV and DAS where as NIV was detected in the culture filtrate of ICR57 (Table 2). There is a general consensus that Fusarium growing on sorghum mainly belongs to Gibberella fujikuroi species complex which comprises of Fusarium species included in section Liseola (Leslie et al. 2005). Members of this group are generally known to produce fumonisins. However, trichothecene production has been associated with isolates of $F$. verticillioides isolated from India and Spain (Ramakrishna et al. 1989; Cantalejo et al. 1999). The EF1 sequence from FM242 had $97 \%$ similarity to $F$. proliferatum and $96 \%$ to $F$. globosum in a BLAST search. Multiple sequence alignment and phylogenetic analysis underlined the similarity of sequence between FM242 and F. proliferatum (Fig. 2). F. globosum is a close relative of $F$. proliferatum and is classified in the Liseola section of Fusarium (Fandohan et al. 2003). AFLP also revealed $\sim 60 \%$ genetic similarity between FM242 and the group of isolates identified as $F$. proliferatum (Fig. 4). Like the rest of the $F$. proliferatum group in this study, the isolate FM242 was also identified as a DON producer. The EF- $1 \alpha$ gene sequence of FM311 which produced microconidia alone when grown on banana leaf 


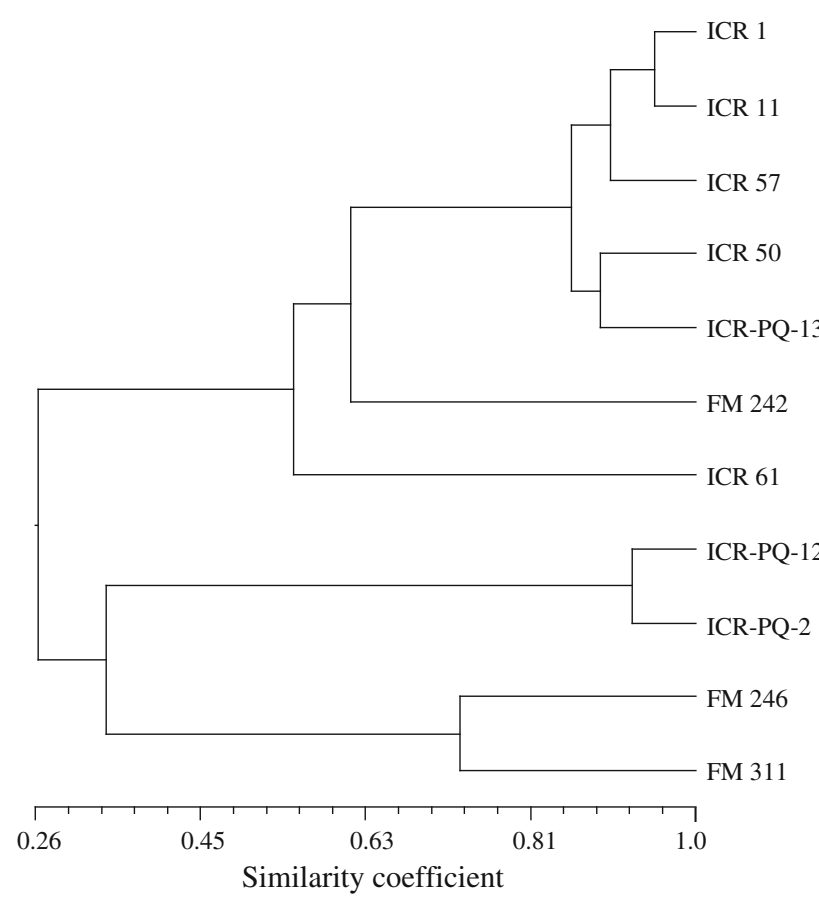

Fig. 4 Cluster analysis derived from AFLP profiles of 11 isolates using 5 primer combinations

agar and the toxins DON and Fus-X in the culture filtrate, was identical to that of $F$. thapsina $(=F$. thapsinum) as assessed while using BLAST and molecular phylogeny analysis [(strong bootstrap support of 89\%) (Table 2, Fig. 2)]. F. thapsinum included in the Liseola section is a known fumonisin producer (Fandohan et al. 2003). ICR61 was identified as $F$. sacchari by multiple sequence alignment, and molecular phylogeny (Fig. 2). The AFLP data also revealed genetic dissimilarity of this isolate from other isolates/species of Fusarium (Fig. 4). As described earlier, this species belongs to the Liseola section which contains fumonisin-producing species of Fusarium. The production of DON by the isolate ICR61 is in agreement with the report of Štyriak et al. (1994), who have isolated F. sacchari capable of synthesizing DON from broiler feed. Morphological features characteristics of the isolates ICRPQ-2 and ICR-PQ-12, when grown on banana leaf agar, resembled that reported for $F$. nelsonii. In NJ analysis, the bootstrap value of these isolates were significantly higher between themselves (99\%) than when compared with F. nelsonii [(63\%) (Fig. 2)]. However the EF-1 $\alpha$ sequence alignment and AFLP agreed completely with the identification of ICR-PQ-2 and ICR-PQ-12 as F. nelsonii, a species of Fusarium which has not hitherto been reported to produce any toxin (Leslie and Summerell 2006). In this study, the isolate ICR-PQ-12 was identified as producer of DON whereas ICR-PQ-2 secreted small amounts of Fus-X along with DON.
Isolate FM246 was identified as $F$. equiseti after comparison of spore morphology and EF1 sequence, which agreed with the grouping of FM246 and $F$. equiseti in a single clade with a bootstrap support of $80 \%$ in the molecular phylogenetic analysis (Table 2, Fig. 2). The isolate FM246 was revealed to be a producer of DON. This is in concordance with the reported production of the trichothecenes DON, DAS and NIV by F. equiseti (Molto et al. 1997; Moss and Thrane, 2004).

\section{Conclusion}

In this paper, we present the first detailed study of Fusarium isolated from India, where we report the presence of different toxigenic Fusarium spp. that are of great importance in understanding the grain mold, a major problem for sorghum in India. This is the primary report from India on identification of trichothecegenic Fusaria where the presence of tri 5 gene has also been demonstrated along with toxin production. In this study, we observed a very strong correlation between the presence of the tri 5 gene and production of trichothecenes by Fusarium isolates, and hence demonstration of the presence of tri 5 gene is indicative of the ability of a Fusarium strain to produce trichothecene. Production of trichothecenes by $F$. nelsonii and $F$. thapsinum is being reported for the first time. The presence of trichothecene-producing strains of Fusarium in Indian sorghum indicates the possibility of large scale contamination of the grains with these toxins and so demands proper screening of food commodities for the detection of these toxins or toxigenic strains of the fungus.

Acknowledgments The project was funded by Department of Biotechnology (DBT). We thank Dr. E. Rati Rao for the kind suggestions and support. S. V. Lincy is grateful to CSIR for providing fellowship. We thank K. Salini, Shalini, Sivaswamy and M. Laxman for technical assistance. We wish to thank Dr. S. G. Prapulla for editing an earlier version of this manuscript.

\section{References}

Adejumo TO, Hettwer U, Karlovsky P (2007) Occurrence of Fusarium species and trichothecenes in Nigerian maize. Int $\mathbf{J}$ Food Microbiol 116:350-357

Altschul SF, Madden TL, Schaffer AA, Zhang JH, Zhang Z, Miller W, Lipman DJ (1997) Gapped BLAST and PSI-BLAST: a new generation of protein database search programs. Nucleic Acids Res 25:3389-3402

Ayalew A, Fehrmann H, Lepschy J, Beck R, Abate D (2006) Natural occurrence of mycotoxins in staple cereals from Ethiopia. Mycopathologia 162:57-63

Bhat RV, Beedu SR, Ramakrishna Y, Munshi KL (1989) Outbreak of trichothecene mycotoxicosis associated with consumption of mould damaged wheat products in Kashmir valley, India. Lancet $7: 35-37$ 
Bhat RV, Shetty PH, Amruth RP, Sudershan RV (1997) A foodborne disease outbreak due to the consumption of moldy sorghum and maize containing fumonisins mycotoxins. J Toxicol-Clin Toxic 35:249-255

Bhavanishankar TN, Shantha T (1987) Natural occurrence of Fusarium toxins in peanut, sorghum and maize from Mysore (India). J Sci Food Agr 40:327-332

Bottalico A, Perrone G (2002) Toxigenic Fusarium species and mycotoxins associated with head blight in small grain cereals in Europe. Eur J Plant Pathol 108:611-624

Cantalejo MJ, Torondel P, Amate L, Carrasco JM, Hernández E (1999) Detection of fusarin C and trichothecenes in Fusarium strains from Spain. J Basic Microb 39:143-153

Desjardins AE, Hohn TM, McCormick SP (1993) Trichothecene biosynthesis in Fusarium species: Chemistry, genetics and significance. Microbiol Rev 57:595-604

Fandohan P, Hell K, Marasas WFO, Wingfield MJ (2003) Infection of maize by Fusarium species and contamination with fumonisin in Africa. Afr J Biotechnol 2:570-579

Geiser DM, Jiménez-Gasco MM, Kang S, Makalowska I, Veeraraghavan N, Ward TJ, Zhang N, Kuldau GA, O'Donnell K (2004) Fusarium-ID v. 1.0: a DNA sequence database for identifying Fusarium. Eur J Plant Pathol 110:473-479

Janardhana GR, Raveesha KA, Shetty HS (1999) Mycotoxin contamination of maize grains grown in Karnataka (India). Food Chem Toxicol 37:863-868

Kumar S, Tamura K, Nei M (2004) MEGA 3: integrated software for molecular evolutionary genetics analysis and sequence alignment. Brief Bioinform 5:150-163

Langseth W, Elen O (1996) Differences between barley, oats and wheat in the occurrence of deoxynivalenol and other trichothecenes in Norwegian grain. J Phytopathol 44:113-118

Larsen JC, Hunt J, Perrin I, Ruckenbauer P (2004) Workshop on trichothecenes with a focus on DON: summary report. Toxicol Lett 153:1-22

Leslie JF, Summerell BA (2006) The Fusarium laboratory manual. 1st eds. Blackwell, Ames

Leslie JF, Zeller KA, Lamprecht SC, Rheeder JP, Marasas WFO (2005) Toxicity, pathogenicity, and genetic differentiation of five species of Fusarium from sorghum and millet. Phytopathology 95:275-283

Lincy SV, Latha R, Chandrashekar A, Manonmani HK (2008) Detection of toxigenic fungi and quantification of type $\mathrm{A}$ trichothecene levels in some food and feed materials from India. Food Control 19:962-966

Martins ML, Martins HM (2001) Determination of deoxynivalenol in wheat based cereals marketed in Portugal. J Food Microbiol 64:1848-1850

Molto GA, Gonzalez HHL, Resnik SL, Gonzalez PA (1997) Production of trichothecenes and zearalenone by isolates of Fusarium spp. from Argentinian maize. Food Addit Contam $14: 263-268$

Moss MO, Thrane U (2004) Fusarium taxonomy with relation to trichothecene formation. Toxicol Lett 53:23-28
Niessen L, Vogel RF (1998) Group specific PCR-detection of potential trichothecene producing Fusarium species in pure cultures and cereal samples. Syst Appl Microbiol 21:618-631

O’Donnel K, Kistler HC, Cigelnik E, Ploetz RC (1998) Multiple evolutionary origins of the fungus causing Panama disease of banana: concordant evidence from nuclear and mitochondrial gene genealogies. Proc Natl Acad Sci 95:2044-2049

Onji Y, Aoki Y, Tani N, Umebayashi K, Kitada Y, Dohi Y (1998) Direct analysis of several Fusarium mycotoxins in cereals by capillary gas chromatography-mass spectrometry. J Chromatogr A 815:59-65

Placinta CM, D'Mello JPF, McDonald AMC (1999) A review of worldwide contamination of cereal grains and animal feed with Fusarium mycotoxins. Anim Feed Sci Tech 78:21-37

Prasad BK, Sahoo DR, Kumar M, Narayan N (2000) Decay of chilli fruits in India during storage. Indian Phytopath 53:42-44

Ramakrishna Y, Bhat RV, Ravindranath V (1989) Production of deoxynivalenol by Fusarium isolates from samples of wheat associated with a human mycotoxicosis outbreak and from sorghum cultivars. Appl Environ Microbiol 55:2619-2620

Rohlf FJ (2000) NTSYS-pc: numerical taxonomy and multivariate analysis system version 2.2. Exeter software: Setauket, New York

Rukmini C, Bhat RV (1978) Occurrence of T-2 toxin in Fusarium infested sorghum from India. J Agr Food Chem 26:647-649

Saharan MS, Naef A, Kumar J, Tiwari R (2007) Characterization of variability among isolates of Fusarium graminearum associated with head scab of wheat using DNA markers. Curr Sci 92:230-234

Schollenberger M, Müller HM, Rüfle M, Suchy S, Plank S, Drochner W (2006) Natural occurrence of 16 Fusarium toxins in grains and feedstuffs of plant origin from Germany. Mycopathologia $161: 43-52$

Sinha KK (1990) Incidence of mycotoxins in maize grains in Bihar State, India. Food Addit Contam 7:55-61

Štyriak I, Čonková E, Böhm J (1994) Occurrence of Fusarium sacchari var. subglutinans and its mycotoxin production ability in broiler feed. Folia Microbiol 39:579-581

Tag AG, Garifullina GF, Peplow AW, Ake C Jr, Phillips TD, Hohn TM, Beremand MN (2001) A novel regulatory gene Tri 10 controls trichothecene toxin production and gene expression. Appl Environ Microbiol 67:5294-5302

Thakur RP, Reddy BVS, Indira S, Rao VP, Navi SS, Yang XB, Ramesh S (2006) Sorghum grain mold. Information Bulletin No. 72. International crops research institute for the semi-arid tropics, Patancheru 502324, Andhra Pradesh, India

Vos P, Hogers R, Bleeker M, van de Lee T, Hornes M, Frijters A, Pot J, Peleman J, Kuiper M, Zabeau M (1995) AFLP: a new technique for DNA fingerprinting. Nucleic Acids Res 23:4407-4414

Wannemacher RJ, Stanley Z, Wiener MD (2000) Trichothecenes mycotoxins. In: Zajtchuk R (ed) Medical aspects of chemical and biological warfare. Department of the Army, Washington, pp 656-670 